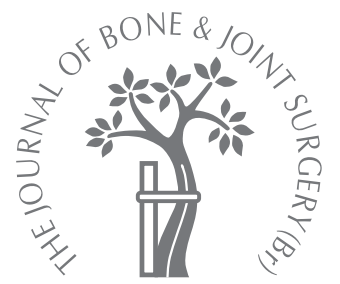

J. Arora,

A. C. Ogden

From Dumfries and Galloway Royal Infirmary, Dumfries, Scotland

\title{
Osteolysis in a surface-cemented, primary, modular Freeman-Samuelson total knee replacement
}

We analysed at a mean follow-up of 7.25 years the clinical and radiological outcome of 117 patients (125 knees) who had undergone a primary, cemented, modular FreemanSamuelson total knee replacement. While the tibial and femoral components were cemented, the patellar component was uncemented. A surface-cementing technique was used to secure the tibial components. A total of 82 knees was available for radiological assessment. Radiolucent lines were seen in 41 knees $(50 \%)$ and osteolytic lesions were seen in 13 knees $(16 \%)$. Asymptomatic, rotational loosening of the patellar implant was seen in four patients and osteolysis was more common in patients with a patellar resurfacing. Functional outcome scores were available for 41 patients ( 41 knees, 35\%) and the mean Western Ontario McMasters Universities score was 77.5 (SD 19.5) and the cumulative survival was $93.4 \%$ at ten years with revision for aseptic loosening as an endpoint. Increased polyethylene wear from modular components, a rotationally-loose patella, and the surface-cementing technique may have contributed to the high rate of osteolysis seen in our study.

The Freeman-Samuelson total knee replacement (TKR) (Sulzer Orthopaedics AG, Baar, Switzerland) is known to have good, predictable long-term results when used in tricompartmental arthritis of the knee. ${ }^{1-3}$ It is a semi-constrained, non-linked prosthesis based on a 'roller in a trough' design. ${ }^{2}$ In the 1980 s, the prosthesis consisted of a polyethylene insert with two pegs which passed through two holes in the stemmed, metal tibial tray. This tibial component was cemented to the proximal surface of the tibia. In the late 1980s, a modular implant was introduced in which the polyethylene insert clipped to the metal tibial tray, which in turn carried a modular, circular cross-section stem and two titanium anchorage pegs.

The aim of this study was to assess the survivorship, and functional and radiological outcomes for a series of modular, primary, cemented Freeman-Samuelson TKRs, in which the tibial component had been secured using a proximal surface cementing technique, with a minimum follow-up of five years.

\section{Patients and Methods}

We identified 117 consecutive patients (125 knees) who underwent a primary TKR by the senior author (ACO) between 1991 and 1998, using a modular Freeman-Samuelson system.
The operation was performed on patients who had severe pain on weight-bearing and at rest, with impaired function and limitation of daily activities. Surgery was only offered if the patient's body mass index (BMI) was $\leq 30 \mathrm{~kg} / \mathrm{m}^{2}$. Implant. The femoral component is made from cobalt-chrome alloy. We used a non-stemmed version, which carries two titanium anchorage pegs. The tibial component is completely modular and comprises a titanium tray with a 50or $80-\mathrm{mm}$ circular cross-sectional stem and two anchorage pegs. The appropriate polyethylene insert was clipped (snap-fit) into the tray at surgery. The polyethylene insert has a single-radius trough which articulates with the femoral component, of the same radius as the trough, from full extension to full flexion. The Freeman-Samuelson TKR also has a highly congruent patellofemoral articulation. The patellar surface is saddle-shaped and articulates with the femoral trochlea in an attempt to minimise maltracking.

Operative procedure. A midline skin incision and a medial parapatellar approach were used. The tibial resection was performed perpendicular to the longitudinal axis of the tibia in both coronal and sagittal planes using an extramedullary guide. The distal femur was resected at $7^{\circ}$ of valgus alignment using an intramedullary guide. The level of the distal femoral resec- 


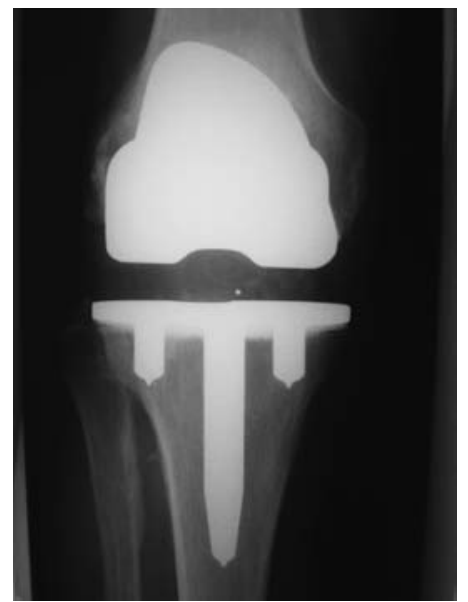

Fig. 1a

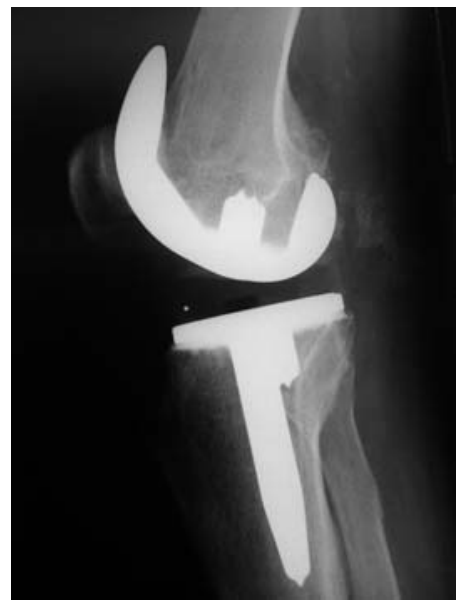

Fig. 1b
Radiographs 4.5 years after total knee replacement undertaken for osteoarthritis in a 68-year-old man a) anteroposterior and b) lateral views. tion, alignment of the leg and soft-tissue balance were confirmed with a tensor instrument.

The proximal tibial surface was drilled to receive two pegs and a stem as tight press fits. The bony surface was then scrubbed and irrigated with pulsatile lavage to open up the cancellous spaces. A proximal (surface) cementing technique was used to secure the tibial tray. This involved placing cement on the bony surface while the holes for the pegs and stem were occluded. The cement was pressurised, partly by finger pressure and partly by impacting the prosthesis. A similar technique was used to fix all the femoral components. Wherever patellar resurfacing was required, an uncemented press-fit all-polyethylene component was used.

Active and passive exercises were started on the first post-operative day. All patients were encouraged to walk with crutches or a walking frame as soon as possible. Six weeks after surgery, a walking stick was advised for a further two months.

Follow-up. Immediate post-operative radiographs (anteroposterior (AP) and lateral views) were taken in the recovery suite. Standing AP, lateral and Merchant's views were taken during the follow-up visits at six weeks, six months, one, two, five and ten years post-operatively (Fig. 1).

The radiographs were evaluated according to the Knee Society evaluation and scoring system ${ }^{4}$ for radiolucencies at the bone-cement interface, wear of polyethylene, change in position of the implants and osteolysis. Osteolysis was defined as an expansile, lucent area measuring at least 5 $\mathrm{mm} \times 5 \mathrm{~mm}$, with loss of trabeculation and a sclerotic rim.

The functional status of the knee was measured with the use of the Western Ontario and McMasters Universities osteoarthritis index (WOMAC) questionnaire, which is a disease-specific measure of pain, stiffness and function. ${ }^{5}$ WOMAC pain and functional scores were transformed to a scale of 0 to 100 .

The survival analysis was calculated by an actuarial lifetable method and survivorship outcomes were reported

\begin{tabular}{ll} 
Table I. Patient details & \\
\hline Mean age in yrs (SD) & 68.7 (8.8) \\
Men:women & $63: 46$ \\
Mean follow-up in yrs (range) & 7.25 (5 to 13) \\
Aetiology (patients) & \\
$\quad$ Osteoarthritis & 87 \\
Rheumatoid arthritis & 23 \\
Psoriasis & 2 \\
Paget's disease & 2 \\
Avascular necrosis & 1 \\
Gout & 1 \\
Total & 109
\end{tabular}

Table II. Indications for revision surgery

\begin{tabular}{ll}
\hline Indication & Number of patients \\
\hline Aseptic loosening & 7 \\
Infection & 6 \\
Anterior knee pain & \\
$\quad$ Unresurfaced patella & 2 \\
$\quad$ After patellar resurfacing & 1 \\
\hline
\end{tabular}

with $95 \%$ confidence intervals (CI). Chi-squared and Fisher exact tests were used for statistical comparison, with the level of significance set at $\mathrm{p} \leq 0.05$.

\section{Results}

Of the 117 patients (125 knees), eight (ten knees) were lost to follow-up within five years of surgery and were excluded from the study, leaving 109 patients (115 knees) for the survivorship analysis. Table I shows the demographic profile of the patients included in this study. No patient who was excluded from the study underwent revision surgery before they were lost to follow-up. By the time of data collection, 24 patients (27 knees) had died. Radiographs of these patients were not available because of the hospital policy of destroying the radiographs of patients who had died. Of the 
Table III. Survival analysis with revision surgery for aseptic loosening and osteolysis as an endpoint

\begin{tabular}{lllllc}
\hline $\begin{array}{l}\text { Interval } \\
\text { (yrs) }\end{array}$ & $\begin{array}{l}\text { Number of } \\
\text { knees at start }\end{array}$ & $\begin{array}{l}\text { Number } \\
\text { of deaths }\end{array}$ & $\begin{array}{l}\text { Number of } \\
\text { knees at risk }\end{array}$ & $\begin{array}{l}\text { Revisions } \\
\text { (aseptic) }\end{array}$ & $\begin{array}{l}\text { Cumulative } \\
\text { survival (\%) }\end{array}$ \\
\hline 0 to 1 & 115 & 3 & 112 & 0 & 100 \\
1 to 2 & 109 & 3 & 106 & 1 & 99.1 \\
2 to 3 & 105 & 1 & 104 & 0 & 99.1 \\
3 to 4 & 102 & 2 & 100 & 1 & 98.1 \\
4 to 5 & 97 & 3 & 94 & 0 & 98.1 \\
5 to 6 & 92 & 2 & 90 & 3 & 94.8 \\
6 to 7 & 74 & 4 & 70 & 1 & 93.4 \\
7 to 8 & 56 & 3 & 53 & 0 & 93.4 \\
8 to 9 & 46 & 2 & 44 & 0 & 93.4 \\
9 to 10 & 34 & 1 & 33 & 0 & 93.4 \\
\hline
\end{tabular}

remaining 85 patients ( 88 knees), six knees which underwent revision surgery because of infection were excluded, leaving 82 knees for radiological assessment.

Survival analysis. Revision surgery was performed in 16 patients. The indications are shown in Table II. The mean interval between index and revision operations was 48 months. Four patients underwent early revision surgery (i.e. within two years of the index operation). Of these, two had deep infection, one had aseptic loosening and early polyethylene wear secondary to valgus malalignment of the tibial component and one, who did not have a patellar resurfacing at the time of the index operation, had anterior knee pain and required resurfacing. Late revision surgery (i.e. two years or more after the index operation) was required in 12 patients, six for aseptic loosening, four for deep infection and two for anterior knee pain (loosening of the patellar component in one and late resurfacing of the patella in the other). For the four patients with deep infection, the infection resulted from haematogenous spread secondary to urinary tract infection in three patients while no obvious cause was found in the fourth.

Revision surgery was undertaken in five of 23 patients with rheumatoid arthritis, two for aseptic loosening, two for anterior knee pain and one for early infection. There was no significant difference in the incidence of overall infection and revision rates between those patients with or without rheumatoid arthritis (chi-squared test; > 0.10).

Out of 115 knees, 87 had patellar resurfacing at the time of the index procedure. At final follow-up, only one had undergone revision surgery because of loosening of the patellar component. Recurrent dislocation of the patella was seen in one patient, who refused further surgery. We did not encounter any extensor mechanism disruption or patellar fracture. Of the seven patients who underwent revision surgery for aseptic loosening, four had rotational loosening of the patellar component, this could be rotated through an arc of $30^{\circ}$ but could not be translated in any direction. The patellar component was also revised in these patients in combination with the tibial and femoral components.

The cumulative ten-year survival of the implant was $93.42 \%$ with revision surgery for aseptic loosening and

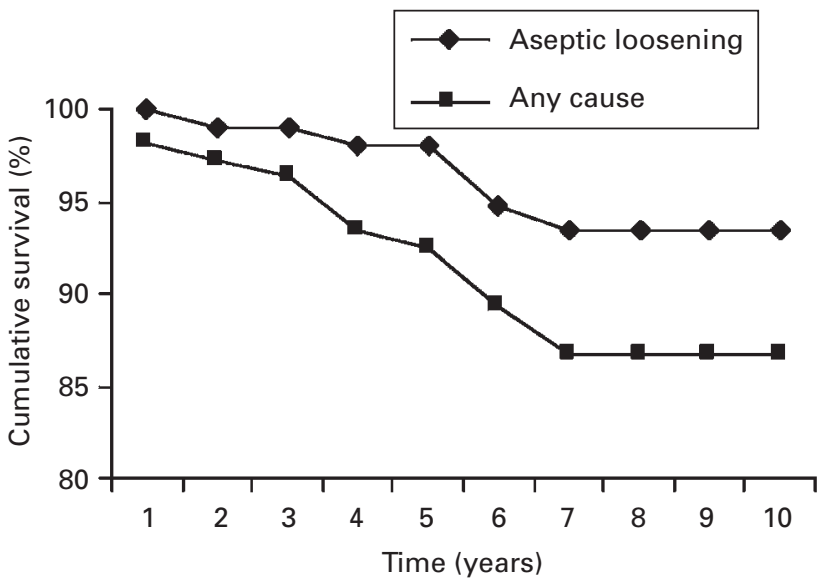

Fig. 2

Survivorship curves showing revision for aseptic loosening and osteoysis and revision for any cause as end-points.

osteolysis as the end-point (95\% CI 85 to 100; Table III). The ten-year survivorship based on revision for any cause was $85 \%$ (Fig. 2).

Radiolucent lines. Radiolucent lines were seen in 41 knees $(50 \%)$. They were more common around the tibial component (37 knees) than the femoral component (ten knees). In order of decreasing frequency, the radiolucent lines were seen around medial femoral condyle ( 32 knees), lateral femoral condyle (24), beneath the anterior tibial tray (21), beneath the posterior tibial tray (15) and beneath the anterior (seven) and posterior (three) flanges of the femoral component.

In 22 knees, the radiolucent lines were incomplete, $<2$ $\mathrm{mm}$ in size, and apparent within two years of surgery. There was no clinical evidence of component migration or loosening in these patients. In 19 knees, the radiolucent lines were complete (present beneath both the medial and lateral parts of the tibial tray) and/or progressive in nature.

Osteolytic lesions. There were 14 osteolytic lesions in 13 knees $(16 \%)$ at the time of the final follow-up. These were seen around the tibial component in 12 knees and around the femoral component in two. The most common site for 
Table IV. Relationship between patellar resurfacing, thickness of the polyethylene insert and the rate of osteolysis

\begin{tabular}{|c|c|c|c|c|c|}
\hline & $\begin{array}{l}\text { Number of patients } \\
\text { with osteolysis }\end{array}$ & $\begin{array}{l}\text { Number of patients } \\
\text { without osteolysis }\end{array}$ & Total & $\begin{array}{l}95 \% \text { confidence } \\
\text { interval }\end{array}$ & $p$ value \\
\hline 6-mm insert & 11 & 38 & 82 & 0.33 to 3.5 & $>0.10$ \\
\hline Without patellar resurfacing & 0 & 5 & \multirow{2}{*}{82} & \multirow{2}{*}{0 to 3.65} & \multirow{2}{*}{0.512} \\
\hline With patellar resurfacing & 19 & 58 & & & \\
\hline
\end{tabular}

an osteolytic lesion was anterior to the tibial stem (nine) followed by the medial tibial condyle (seven).

An enlarging osteolytic lesion (two cases) and progressive radiolucent line (one) beneath the tibial implant were seen in three patients with aseptic loosening of the femoral component. During revision surgery in these patients, the tibial components were also revised, despite being found to be firmly fixed. There was no correlation $(p>0.10)$ between the thickness of the polyethylene insert and the incidence of osteolysis or radiolucent lines. Osteolysis and radiolucent lines were more common in patients with a patellar resurfacing (Table IV); this difference was not, however, statistically significant $(95 \%$ CI 0 to $3.65 ; \mathrm{p}=$ 0.512).

Functional outcome. At the time of data collection, a WOMAC questionnaire was sent to 57 patients (58 knees), who were mentally competent and still had their primary TKR in place; 41 patients (41 knees, 35\%) responded. The mean WOMAC score was 77.5 (SD 19.5) and the mean pain score was 83.2 (SD 20.6), indicating mild pain with some activities. The mean function score was 76 (SD 20.6), also indicating mild limitation of most activities.

\section{Discussion}

Various studies ${ }^{2,3}$ have reported a satisfactory survivorship and clinical outcome after a Freeman-Samuelson TKR, including an analysis of the Swedish knee arthroplasty register for 2695 primary Freeman-Samuelson TKRs, which revealed a survivorship of $96 \%$ at ten years with aseptic loosening as the end-point. ${ }^{1}$ However, in these studies, the type of implant (modular or monobloc) and the cementing technique used (cemented, uncemented, and proximal/full cementation) were not uniform. In our study the modular, cemented primary Freeman-Samuelson TKR knee replacements had a survivorship of $93.4 \%$ at ten years because of aseptic loosening and a satisfactory clinical outcome based upon WOMAC scores. However, a high rate of osteolysis $(14 \%)$ and radiolucent lines $(50 \%)$ was found. We believe that the actual incidence of radiolucent lines may be even higher. The radiographs were not taken after screening and orientating the $\mathrm{x}$-ray beam parallel to the tibial tray and may, therefore, have resulted in an underestimation of the incidence of the radiolucent lines. To our knowledge, the medium-term survivorship, with a clinical and radiological analysis of the cemented, modular Freeman-Samuelson TKR, has not to date been reported in the English literature.
Osteolysis after TKR is well recognised and its incidence depends on factors such as implant design, the use of cement and length of follow-up. ${ }^{6,7}$ It is less common with highly conforming implant designs such as the Freeman-Samuelson, because of a low polyethylene wear rate. ${ }^{8}$ The introduction of modular implants has been shown to result in an accelerated polyethylene wear and an increase in the rate of osteolysis, being first reported in a retrieval study by Engh, Dwyer and Hanes. ${ }^{9}$ Wasielewski et $\mathrm{al}^{10}$ and Robinson et $\mathrm{al}^{11}$ also showed that microscopic, backside wear of modular, non-rotating tibial components can generate a significant quantity of polyethylene particles. In our study, an increased backside wear between the matt surface of the tibial tray and the polyethylene could be one of the factors leading to the increased rate of osteolysis.

In 2002, Valdivia et $\mathrm{al}^{12}$ reported better results for uncemented than cemented patellar replacements in 172 patients with Freeman-Samuelson patellar and femoral components. They found a low incidence of patellar maltracking in the uncemented group which was attributed to the ability of the press-fit implant to rotate and centre itself in the trochlear groove as the knee moved through its flexion/extension cycle. This assertion was based on the finding of an asymptomatic, rotationally loose, press-fit patellar component during revision surgery for tibial component loosening. Asymptomatic rotational loosening of FreemanSamuelson patellar components was also noticed in four patients in our study and in three patients in the study by Kulkarni et al. ${ }^{13}$ It is difficult to confirm the rate of asymptomatic rotational loosening of the patella but, based on our study, it could be more than $50 \%$ (four of seven patients). The movement between the non-articular patellar surface and the patellar bone could lead to an increase in polyethylene wear and may have contributed to the increased rate of osteolysis in our study. We found that the osteolysis was more common in patients with, rather than without a patellar resurfacing although the difference was not statistically significant. As our study was retrospective we could not confirm increased wear on the non-articular surface by retrieval analysis of the patellar components. Kulkarni et $\mathrm{al}^{13}$ compared the ten-year outcome of 124 Freeman-Samuelson TKRs with an all-polyethylene pressfit patellar resurfacing with 143 Freeman-Samuelson TKRs without patellar resurfacing. The rate of aseptic loosening was higher in the patellar resurfacing group (16 of 124) as compared with the non-resurfacing group (11 of 143). 
However, the rate of osteolysis between the two groups was not compared. ${ }^{13}$

In the literature, the rate of osteolysis in cemented TKRs has been low despite retrieval studies which have documented substantial wear of the tibial polyethylene. ${ }^{7}$ In contrast, the high rate of osteolysis leading to revision surgery in uncemented TKRs has been well documented. ${ }^{14}$ This difference could be because of variables such as implant design, operative technique, patient-related factors and the presence of cement. It is thought that the cement mantle reduces the effective joint space by preventing the joint fluid, laden with polyethylene wear particles and soluble factors such as interleukins and cytokines, access to the tibial metaphyseal bone, thereby reducing the osteolysis. However, debate continues whether the tibial stem should be cemented at the same time as the undersurface of tibial tray. Proponents of surface cementing argue that adequate stability is achieved with decreased metaphyseal bone loss evident at revision, and the potential for stress shielding is reduced. Although biomechanical studies on cadaver specimens have shown no difference in the stability of the implants secured either by a surface- or fully-cemented technique, ${ }^{15,16}$ clinical studies have reported a high rate of aseptic loosening with surface cementing. ${ }^{17}$ It is thought that the presence of radiolucent lines beneath the tibial tray would not only prejudice the fixation of a surface-cemented tibial implant, but might also provide a portal of entry for the joint fluid, and all its contents, to a large area of metaphyseal bone around the uncemented stem; this might lead to osteolysis and loosening. ${ }^{7}$ In a study of 207 FreemanSamuelson TKRs, in which the tibial components were fixed with a surface-cementing technique, Smith et $\mathrm{al}^{3}$ reported that 12 patients, who had severe polyethylene wear because of femoral component loosening and subsidence, developed tibial osteolysis. Of these, nine also had associated radiolucent lines under the tibial implant. The authors concluded that surface cementing was only satisfactory in low-wear situations and was associated with an increased rate of osteolysis and polyethylene wear. ${ }^{3}$ Their study included both modular and monobloc implants, with no distinction being made between the two designs. We also noticed a progressive radiolucency and osteolysis around the tibial component of three patients who had aseptic loosening of the femoral component.

We conclude that, although a modular Freeman Samuelson TKR has a satisfactory medium-term survivorship and clinical outcome, it is associated with a high rate of osteolysis and radiolucent lines. Wear from a rotationally loose, all-polyethylene press-fit patellar component and the use of surface cementing may have contributed to the increased osteolysis seen in our study.

\section{Supplementary Material}

$\because$ A further opinion by Mr Gareth Scott is available with the electronic version of this article on our website at www.jbjs.org.uk

No benefits in any form have been received or will be received from a commercial party related directly or indirectly to the subject of this article.

\section{References}

1. Robertsson O, Scott G, Freeman MAR. Ten-year survival of the cemented Freeman-Samuelson primary knee arthroplasty: data from the Swedish Knee Arthroplasty register and the Royal London Hospital. J Bone Joint Surg [Br] 2000;82-B:506-7.

2. Freeman MAR, Swanson SAV, Todd RC. Total replacement of the knee using the Freeman-Swanson knee prosthesis. Clin Orthop 2003;416:4-21.

3. Smith S, Naima VSN, Freeman MAR. The natural history of tibial radiolucent lines in a proximally cemented stemmed total knee arthroplasty. J Arthroplasty 1999;14: 3-8.

4. Ewald FC. The Knee Society total knee arthroplasty roentgenographic evaluation and scoring system. Clin Orthop 1989;248:9-12

5. Bellamy N, Buchanan WW, Goldsmith CH, Campbell J, Stitt LW. Validation study of WOMAC: a health status instrument for measuring clinically important patient relevant outcomes to antirheumatic drug therapy in patients with osteoarthritis of the hip or knee. J Rheumato/ 1988;15:1833-40.

6. Harris WH. Wear and periprosthetic osteolysis: the problem. Clin Orthop 2001;393: 66-70

7. Schmalzried TP, Callaghan JJ. Wear in total hip and knee replacements. J Bone Joint Surg [Am] 1999;81-A:115-36.

8. Bartel DL, Bicknell VL, Wright TM. The effect of conformity, thickness and material on stresses in ultra-high molecular weight components for total joint replacement. J Bone Joint Surg [Am] 1986;68-A:1041-51.

9. Engh CA, Dwyer KA, Hanes CK. Polyethylene wear of metal backed tibial components in total and uni-compartmental knee prosthesis. J Bone Joint Surg [Br] 1992; 74-B:9-17.

10. Wasielewski RC, Parks N, Williams I, et al. Tibial insert undersurface as a contributing source of polyethylene wear debris. Clin Orthop 1997;345:53-9.

11. Robinson EJ, Mulliken BD, Bourne RB, Rorabeck CH, Alvarez C. Catastrophic osteolysis in total knee replacement: a report of 17 cases. Clin Orthop 1995:321: 98-105.

12. Valdivia GG, Dunbar MJ, Jenkinson RJ, et al. Pressfit versus cemented all polyethylene patellar component: midterm results. J Arthroplasty 2002;17:20-5.

13. Kulkarni SK, Freeman MAR, Poal-Manresa JC, Ascencio J, Rodriguez JJ. The patellofemoral joint in total knee arthroplasty: is the design of the trochlea the critical factor? J Arthroplasty 2000;15:424-9.

14. Rand JA, Trousdale RT, Ilstrup DM, Harmsen WS. Factors affecting the durability of primary total knee prosthesis. J Bone Joint Surg [Am] 2003;85-A:259-65.

15. Peters CL, Craig MA, Mohr RA, Bachus KN. Tibial component fixation wth cement: full-versus surface-cementation techniques. Clin Orthop 2003:409:158-68.

16. Bourgeault ST, Chareancholvanich $\mathbf{K}$, Lew $\mathbf{W}$, et al. Does a central stem effect bone strain and the stability of a cemented tibial tray in primary and revision TKA? Trans Orthop Res Soc 1997;22:635.

17. Sharkey PF, Hozack WJ, Rothman RH, Shastri S, Jacoby SM. Why are total knee arthroplasties failing today? Clin Orthop 2002;404:7-13. 